\title{
Bivalves and gastropods of Tubbataha Reefs Natural Park, Philippines
}

\author{
Roger G. Dolorosa ${ }^{1 *}$, Rafael M. Picardal ${ }^{1}$ and Segundo F. Conales Jr. ${ }^{2}$ \\ 1 College of Fisheries and Aquatic Sciences, Western Philippines University-Puerto Princesa Campus, Sta. Monica, Puerto Princesa City, Philippines \\ 2 Tubbataha Management Office, 5300 Puerto Princesa City, Philippines \\ * Corresponding author. E-mail: rogerdolorosa@yahoo.com
}

\begin{abstract}
: in this study of marine bivalves and gastropods of Tubbataha Reefs Natural Park (TRNP), Palawan, Philippines, we photo-document 96 species, including 17 species of bivalves (in seven families), and 79 species of gastropods (in 29 families). Of these, 64 species (eight bivalves and 56 gastropods species) were new recorded from the park. The list also includes the seven species of giant clams which are protected under the Convention on International Trade in Endangered Species of Wild Fauna and Flora (CITES) and three nationally protected gastropod species (Cassis cornuta, Charonia tritonis and Tectus niloticus). Thirty-six species that were previously reported at the park were not found in this study, which suggests that there are at least 132 species of gastropods and bivalves at TRNP. Our observations were limited at intertidal and shallow subtidal reefs in the park, suggesting that the number of species in the list may rise with an extensive survey.
\end{abstract}

Key words: Mollusca; Bivalvia; Gastropoda; species inventory; Tubbataha Reefs Natural Park; Philippines

\section{INTRODUCTION}

The Tubbataha Reefs Natural Park (TRNP) in the middle of the Sulu Sea, the Philippines, is rich in biodiversity. in 1993 the park was named by the United Nations, Educational, Scientific and Cultural Organization (UNESCO) as a World Heritage Site, and research has been done to document and protect the rich flora and fauna. Currently there are ca. 400 species of corals ( $85 \%$ of known coral species in the Philippines), nearly 700 species of fish, two species of marine turtles, seven species of breeding seabirds, 13 species of cetaceans, 79 species of algae and 10 species of seagrass (Dygico 2006; Songco 2012; TMO 2013).

Although many taxa have been inventoried in TRNP, not much is known about the park's molluscan fauna, which Yamaguchi (1996) first documented. Other studies did not extensively focus on mollusks but reported a few species (see Estacion et al. 1993; Dolorosa and Schoppe 2005; Dolorosa et al. 2011; Dolorosa and Jontila 2012; Dolorosa et al. 2013). We include previously published data, along our new observations made between May 2005 and June 2010, to provide an updated list of the mollusks of TRNP. Given such meager information, this list of mollusks in TRNP is hoped to complement all other information about the rich and undocumented diversity in the Islands of Palawan, Philippines.

\section{MATERIALS AND METHODS}

This species list of mollusks of TRNP is based on photos made within TRNP, Palawan, Philippines ( $08^{\circ} 43^{\prime}-08^{\circ} 57^{\prime} \mathrm{N}$ and $119^{\circ} 48^{\prime}-120^{\circ} \mathrm{O} 3^{\prime} \mathrm{E}$ ), during annual biodiversity monitoring between May 2005 and June 2010. The TRNP is a no-take zone, and for this reason, no voucher specimens were collected. Photos of bivalves and gastropods were identified with the aid of various reference materials (Springsteen and Leobrera 1986; Aubry 1999; Abbott and Dance 2000; Laureta 2008; Terryn 2007; Poppe 2008a; Poppe 2008b; Poppe 2010a; Poppe 2010b). The species' taxonomy, including the species reported in prior publications, is according to the World Register of Marine Species (WoRMS 2014). Species are alphabetical within families. Also included in this list of species are those previously published in the literature but not encountered in our study.

\section{RESULTS}

We photo-document 96 species of mollusks from TRNP; 17 of these are bivalves belonging to seven families (Table 1; Figures 1 and 2). We newly record eight bivalves from the park (Table 1). We observed 79 species of gastropods, and 56 of these are new records at TRNP (Table 2; Figures 3-7).

\section{DISCUSSION}

The total number of species (96 species) recorded in this study is much higher compared with the previous record of Yamaguchi (1996) (Tables 1 and 2). However, 36 of the previously reported species were not found, implying that there are at least 132 species of gastropods and bivalves in TRNP.

Among the recorded species, seven bivalves (Tridacninae) are CITES protected (CITES 2014) and three gastropod species (Charonia tritonis, Tectus niloticus, and Cassis cornuta) are protected in the Philippines (Dolorosa and Jontila 2012; Dolorosa et al. 2013). These large mollusks species have high economic, ecological, aesthetic and scientific importance but are becoming rare in many parts of the country; thus, TRNP 
can serve as significant seed source of these valuable species.

The number of species of gastropods and bivalves recorded from such a rich and protected area within the Coral Triangle, the world's center of marine biodiversity, is very low considering that Springsteen and Leobrera (1986) reported more than 1,60o species of mollusks from the Philippines. Previous studies were limited to intertidal areas (e.g., Batomalaque et al. 2010, who had reported 100 species). Our study was undertaken in intertidal and shallow subtidal coral reefs and sandy habitats and focused mainly on largeshelled species (over ca. $2 \mathrm{~cm}$ ) and neglected smaller. The deeper water sandy areas inside the lagoon and the $c a .1000$ $\mathrm{m}$ abyss surrounding the reefs remain unexplored. A deep sea expedition in Panglao, Bohol, Philippines found about 6,0oo species (Richer de Forges et al. 2009), which include new species and new records for the Philippines. We anticipate that continued exploration of TRNP in both shallow and deep water habitats and with small-bodied species targeted may reveal new records for the park, Palawan, and the Philippines.

\section{ACKNOWLEDGMENTS}

This study is an offshoot of several years' of annual monitoring within TRNP, with funding from the Tubbataha Management Office, Conservation International, and World Wildlife Fund for Nature. We thank U. Aubry and J. Sarino for confirming some identifications. L. Creencia read an early draft of the manuscript. The comments of the two anonymous reviewers are greatly acknowledged.

\section{LITERATURE CITED}

Abbott, R.T. and S.P. Dance. 200o. Compendium of Seashells. Odyssey Publishing, USA. $411 \mathrm{pp}$.

Aubry, U. 1999. Nuove Terebre e Antichi Versi (New Terebras and Ancient Verses). L'informatore Piceno Ed., Ancona, 47 pp.

Batomalaque, G.A., B.G.P. Arce, M.B.M. Hernandez and I.K.C. Fontanilla. 2010. Survey and spatial distribution of shoreline malacofauna in Grande Island, Subic Bay. Philippine Journal of Science 139 (2): 149-159 (http://philjournalsci.dost.gov.ph/ vol139no2/volume_139_No2.html).

CITES. 2014. Convention on International Trade in Endangered Species of Wild Fauna and Flora. Appendices I, II and III. Available at http:// www.cites.org. Captured on 6 August 2014.

Dolorosa, R.G., S. F. Conales and N.A. Bundal. 2011. Initial stock assessment of Terebra maculata (Gastropoda: Terebridae) in Tubbataha Reefs Natural Park, Palawan, Philippines. The Philippine Scientist 48: 85-99 (http://www.philjol.info/philjol/ index.php/PSCI/article/view/2482/2125).

Dolorosa, R.G., S.F. Conales and N.A. Bundal. 2013. Status of Horned Helmet Cassis cornuta in Tubbataha Reefs Natural Park, and its trade in Puerto Princesa City, Philippines. Atoll Research Bulletin 595:1-17 (doi: 10.5479/si.00775630.595).

Dolorosa, R.G. and J.B.S. Jontila. 2012. Notes on common macrobenthic reef invertebrates of Tubbataha Reefs Natural Park, Philippines. Science Diliman 24: 1-11 (http://journals.upd.edu.ph/index.php/ sciencediliman/article/viewFile/3733/3434).

Dolorosa, R.G. and S. Schoppe. 2005. Focal benthic mollusks (Mollusca: Bivalvia and Gastropoda) of selected sites in Tubbataha Reefs National Marine Park, Palawan, Philippines. Science Diliman 17: 1-10 (http://journals.upd.edu.ph/index.php/sciencediliman/article/ viewFile/1/11)

Table 1. Bivalve species from Tubbataha Reefs Natural Park, Philippines;

$=$ recorded; $\mathrm{O}=$ previously recorded but not found this study; $\star=$ new ecords, this study.

\begin{tabular}{|c|c|c|c|c|c|c|}
\hline Family & Species & $\begin{array}{c}\text { Yamaguchi } \\
1996\end{array}$ & $\begin{array}{c}\text { Estacion et al. } \\
1993\end{array}$ & $\begin{array}{c}\text { Dolorosa } \\
\text { and Schoppe } \\
2005\end{array}$ & $\begin{array}{l}\text { Dolorosa and } \\
\text { Jontila } 2012\end{array}$ & This study \\
\hline Arcidae & Anadara sp. & O & & & & \\
\hline \multirow[t]{8}{*}{ Cardiidae } & Fragum fragum (Linnaeus, 1758) & O & & & & \\
\hline & Hippopus hippopus (Linnaeus, 1758) & $\bullet$ & $\bullet$ & $\bullet$ & & $\bullet$ \\
\hline & Hippopus porcellanus Rosewater, 1982 & & $\bullet$ & & $\bullet$ & $\bullet$ \\
\hline & Tridacna crocea Lamarck, 1819 & $\bullet$ & $\bullet$ & $\bullet$ & $\bullet$ & $\bullet$ \\
\hline & Tridacna derasa (Röding, 1798) & & $\bullet$ & & & $\bullet$ \\
\hline & Tridacna gigas (Linnaeus, 1758) & & $\bullet$ & & & $\bullet$ \\
\hline & Tridacna maxima Röding, 1798 & $\bullet$ & $\bullet$ & $\bullet$ & $\bullet$ & $\bullet$ \\
\hline & Tridacna squamosa Lamarck, 1819 & & $\bullet$ & $\bullet$ & $\bullet$ & $\bullet$ \\
\hline \multirow[t]{3}{*}{ Lucinidae } & Fimbria fimbriata (Linnaeus, 1758) & & & & & $\star$ \\
\hline & Codakia tigerina (Linnaeus, 1758) & & & & & $\star$ \\
\hline & Ctena bella (Conrad, 1837) & O & & & & \\
\hline \multirow[t]{2}{*}{ Mesodesmatidae } & Atactodea striata (Gmelin, 1791) & $\bullet$ & & & & $\bullet$ \\
\hline & Davila plana (Hanley, 1843) & O & & & & \\
\hline Mytilidae & Modiolus philippinarum (Hanley, 1843) & O & & & & \\
\hline \multirow[t]{2}{*}{ Ostreidae } & Lopha cristagalli (Linnaeus, 1758) & & & & & $\star$ \\
\hline & Saccostrea mordax (Gould, 1850) & & & & & $\star$ \\
\hline Pinnidae & Pinna muricata Linnaeus, 1758 & O & & & & \\
\hline Psammobiidae & Asaphis violascens (Forsskål in Niebuhr, 1775) & & & & & $\bullet$ \\
\hline \multirow[t]{3}{*}{ Tellinidae } & Tellina perna Spengler, 1798 & O & & & & \\
\hline & Tellina scobinata Linnaeus, 1758 & & & & & $\star$ \\
\hline & Tellina sp. & & & & & $\star$ \\
\hline \multirow[t]{3}{*}{ Veneridae } & Gafrarium pectinatum (Linnaeus, 1758) & O & & & & \\
\hline & Lioconcha castrensis (Linnaeus, 1758) & & & & & $\star$ \\
\hline & Periglypta puerpera (Linnaeus, 1771) & & & & & $\star$ \\
\hline Total & & 12 & 7 & 4 & 4 & 17 \\
\hline
\end{tabular}


Table 2. Gastropod species from Tubbataha Reefs Natural Park, Philippines; $\bullet=$ recorded; $O=$ previously recorded but not found this study; $\star=$ new ecords, this study.

\begin{tabular}{|c|c|c|c|c|c|c|}
\hline Family & Species & $\begin{array}{c}\text { Yamaguchi } \\
1996\end{array}$ & $\begin{array}{c}\text { Estacion et al. } \\
1993\end{array}$ & $\begin{array}{c}\text { Dolorosa } \\
\text { and Schoppe } \\
2005\end{array}$ & $\begin{array}{l}\text { Dolorosa and } \\
\text { Jontila } 2012\end{array}$ & This study \\
\hline Angariidae & Angaria delphinus (Linnaeus, 1758) & & & & & $\star$ \\
\hline \multirow[t]{3}{*}{ Bursidae } & Tutufa bubo (Linnaeus, 1758) & & & & $\bullet$ & $\bullet$ \\
\hline & Bursa lamarckii (Deshayes,1853) & & & & & $\star$ \\
\hline & Bursa granularis (Röding, 1798) & & & & & $\star$ \\
\hline Cancellariidae & Scalptia sp. & O & & & & \\
\hline \multirow[t]{2}{*}{ Cassidae } & Cassis cornuta (Linnaeus, 1758) & $\bullet$ & & & & $\bullet$ \\
\hline & Casmaria ponderosa (Gmelin, 1791) & & & & & $\star$ \\
\hline \multirow[t]{4}{*}{ Cerithiidae } & Cerithium nodulosum Bruguière, 1792 & & & & & $\star$ \\
\hline & Cerithium sp. & O & & & & \\
\hline & Cerithium rostratum A. Adams in G.B. Sowerby II, 1855 & O & & & & \\
\hline & Pseudovertagus aluco (Linnaeus, 1758) & O & & & & \\
\hline Colubrariidae & Colubraria muricata (Lightfoot, 1786) & & & & & $\star$ \\
\hline \multirow[t]{2}{*}{ Columbeliidae } & Euplica scripta (Lamarck, 1822) & O & & & & \\
\hline & Pyrene flava (Bruguière, 1789) & O & & & & \\
\hline \multirow[t]{21}{*}{ Conidae } & Conus arenatus Hwass in Bruguière, 1792 & O & & & & \\
\hline & Conus coronatus Gmelin, 1791 & O & & & & \\
\hline & Conus distans Hwass in Bruguière, 1792 & & & & & $\star$ \\
\hline & Conus ebraeus Linnaeus, 1758 & & & & & $\star$ \\
\hline & Conus eburneus Hwass in Bruguière, 1792 & & & & & $\star$ \\
\hline & Conus imperialis Linnaeus, 1758 & & & & & $\star$ \\
\hline & Conus leopardus (Röding, 1798) & & & & & $\star$ \\
\hline & Conus litteratus Linnaeus, 1758 & $\bullet$ & & & & $\bullet$ \\
\hline & Conus lividus Hwass in Bruguière, 1792 & & & $\bullet$ & $\bullet$ & $\bullet$ \\
\hline & Conus marmoreus Linnaeus, 1758 & & & & & $\star$ \\
\hline & Conus miles Linnaeus, 1758 & & & $\bullet$ & & $\bullet$ \\
\hline & Conus pulicarius Hwass in Bruguière, 1792 & O & & & & \\
\hline & Conus sp. 1 & O & & & & \\
\hline & Conus sp. 2 & & & O & & \\
\hline & Conus sponsalis Hwass in Bruguière, 1792 & O & & & & \\
\hline & Conus stercusmuscarum Linnaeus, 1758 & & & & & $\star$ \\
\hline & Conus striatus Linnaeus, 1758 & & & & & $\star$ \\
\hline & Conus tessulatus Born, 1778 & $\bullet$ & & & & $\bullet$ \\
\hline & Conus planorbis Born, 1778 & O & & & & \\
\hline & Conus pulicarius Hwass in Bruguière, 1792 & & & & & $\bullet$ \\
\hline & Conus virgo Linnaeus, 1758 & $\bullet$ & & $\bullet$ & & $\bullet$ \\
\hline Costellariidae & Vexillum exasperatum (Gmelin, 1791) & O & & & & \\
\hline \multirow[t]{10}{*}{ Cypraeidae } & Cypraea tigris Linnaeus, 1758 & & $\bullet$ & $\bullet$ & $\bullet$ & $\bullet$ \\
\hline & Esoraria erosa (Linnaeus, 1758) & & & & & $\star$ \\
\hline & Lyncina lynx (Linnaeus, 1758) & & & & & $\star$ \\
\hline & Lyncina vitellus (Linnaeus, 1758) & & & & & $\star$ \\
\hline & Mauritia arabica (Linnaeus, 1758) & & & & & $\star$ \\
\hline & Monetaria annulus (Linnaeus, 1758) & $\bullet$ & & $\bullet$ & & $\bullet$ \\
\hline & Monetaria moneta (Linnaeus, 1758) & $\bullet$ & & $\bullet$ & & $\bullet$ \\
\hline & Monetaria caputserpentis (Linnaeus, 1758) & & & & & $\star$ \\
\hline & Ovatipsa chinensis (Gmelin, 1791) & & & & & $\star$ \\
\hline & Talparia talpa (Linnaeus, 1758) & & & & & $\star$ \\
\hline \multirow[t]{2}{*}{ Fasciolariidae } & Latirolagena smaragdulus (Linnaeus, 1758) & & & & & $\star$ \\
\hline & Pleuroploca trapezium (Linnaeus, 1758) & & & • & & • \\
\hline Haliotidae & Haliotis asinina Linnaeus, 1758 & & & & & $\star$ \\
\hline Hipponicidae & Cheilea cepacea (Broderip, 1834) & & & & & $\star$ \\
\hline Littorinidae & Littoraria scabra (Linnaeus, 1758) & & & & & $\star$ \\
\hline \multirow[t]{3}{*}{ Mitridae } & Mitra mitra (Linnaeus, 1758) & & & & & $\star$ \\
\hline & Mitra papalis (Linnaeus, 1758) & & & O & & \\
\hline & Neocancilla papilio (Link, 1807) & O & & & & \\
\hline \multirow[t]{2}{*}{ Muricidae } & Chicoreus brunneus (Link, 1807) & & & & & $\star$ \\
\hline & Drupa ricinus (Linnaeus, 1758) & & & & & $\star$ \\
\hline
\end{tabular}


Table 2. Continued.

\begin{tabular}{|c|c|c|c|c|c|c|}
\hline Family & Species & $\begin{array}{c}\text { Yamaguchi } \\
1996\end{array}$ & $\begin{array}{c}\text { Estacion et al. } \\
1993\end{array}$ & $\begin{array}{c}\text { Dolorosa } \\
\text { and Schoppe } \\
2005 \\
\end{array}$ & $\begin{array}{l}\text { Dolorosa and } \\
\text { Jontila } 2012\end{array}$ & This study \\
\hline & Drupella cornus (Röding, 1798) & & & & & $\star$ \\
\hline & Drupella margariticola (Broderip, 1833) & ○ & & & & \\
\hline & Drupina grossularia (Röding, 1798) & & & & & $\star$ \\
\hline \multirow[t]{4}{*}{ Nassariidae } & Nassarius albescens (Dunker, 1846) & ○ & & & & \\
\hline & Nassarius coronatus (Bruguière, 1789) & & & & & $\star$ \\
\hline & Nassarius gaudiosus (Hinds, 1844) & O & & & & \\
\hline & Nassarius papillosus (Linnaeus, 1758) & & & & & $\star$ \\
\hline \multirow[t]{2}{*}{ Naticidae } & Notocochlis gualteriana (Récluz, 1844) & & & & & $\star$ \\
\hline & Mammilla sebae (Récluz, 1844) & & & & & $\star$ \\
\hline \multirow[t]{2}{*}{ Neritidae } & Nerita polita (Linnaeus, 1758 & & & & & $\star$ \\
\hline & Smaragdia sp. & O & & & & \\
\hline \multirow[t]{3}{*}{ Olividae } & Oliva annulata Gmelin, 1791 & & & & & $\star$ \\
\hline & Oliva miniacea (Röding, 1798) & & & & & $\star$ \\
\hline & Oliva reticulata (Röding, 1798) & & & & & $\star$ \\
\hline Personidae & Distorsio anus (Linnaeus, 1758) & & & & & $\star$ \\
\hline Pyramidellidae & sp. 1 & O & & & & \\
\hline Ranellidae & Charonia tritonis (Linnaeus, 1758) & & & & & $\star$ \\
\hline \multirow[t]{12}{*}{ Strombidae } & Canarium labiatum (Röding, 1798) & & & & & $\star$ \\
\hline & Canarium mutabile (Swainson, 1821) & O & & & & \\
\hline & Canarium urceus (Linnaeus, 1758) & O & & & & \\
\hline & Conomurex luhuanus (Linnaeus, 1758) & $\bullet$ & & & $\bullet$ & $\bullet$ \\
\hline & Euprotomus bulla (Röding, 1798) & & & & & $\star$ \\
\hline & Gibberulus gibberulus gibbosus (Röding, 1798) & & & & & $\star$ \\
\hline & Harpago chiragra (Linnaeus, 1758) & & & $\bullet$ & $\bullet$ & • \\
\hline & Lambis lambis (Linnaeus, 1758) & $\bullet$ & & & & $\bullet$ \\
\hline & Lambis scorpius (Linnaeus, 1758) & & & & & $\star$ \\
\hline & Lambis sp. & & & O & & \\
\hline & Lambis sowerbyi (Mörch, 1877) & & & & & $\star$ \\
\hline & Lentigo lentiginosus (Linnaeus, 1758) & & & & & $\star$ \\
\hline \multirow[t]{4}{*}{ Tegulidae } & Tectus fenestratus (Gmelin, 1791) & & & & & $\star$ \\
\hline & Tectus niloticus (Linnaeus, 1767) & & & $\bullet$ & $\bullet$ & $\bullet$ \\
\hline & Tectus pyramis (Born, 1778) & & & $\bullet$ & $\bullet$ & $\bullet$ \\
\hline & Trochus maculatus Linnaeus, 1758 & & & $\bullet$ & & ○ \\
\hline \multirow[t]{12}{*}{ Terebridae } & Hastula lanceata (Linnaeus, 1767) & & & & & $\star$ \\
\hline & Hastula solida (Deshayes, 1857) & O & & & & \\
\hline & Myurella affinis (Gray, 1834) & $\bullet$ & & & & $\bullet$ \\
\hline & Oxymeris areolata (Link, 1807) & & & & & $\star$ \\
\hline & Oxymeris crenulata (Linnaeus, 1758) & & & & & $\star$ \\
\hline & Oxymeris dimidiata (Linnaeus, 1758) & & & & & $\star$ \\
\hline & Oxymeris felina (Dillwyn, 1817) & & & & & $\star$ \\
\hline & Oxymeris maculata (Linnaeus, 1758) & & & & & $\star$ \\
\hline & Terebra argus Hinds, 1844 & O & & & & \\
\hline & Terebra casta Hinds, 1844 & O & & & & \\
\hline & Terebra guttata (Röding, 1798) & $\bullet$ & & & & - \\
\hline & Terebra subulata (Linnaeus, 1767) & $\bullet$ & & & & $\bullet$ \\
\hline \multirow[t]{2}{*}{ Tonnidae } & Malea pomum (Linnaeus, 1758) & & & & & $\star$ \\
\hline & Tonna canaliculata (Linnaeus, 1758) & & & & & $\star$ \\
\hline Turbinellidae & Vasum turbinellus (Linnaeus, 1758) & ○ & & & & • \\
\hline Turbinidae & Turbo chrysostomus Linnaeus, 1758 & & & $\bullet$ & $\bullet$ & $\bullet$ \\
\hline Total & & 37 & & 15 & 8 & 79 \\
\hline
\end{tabular}


Dygico, M.P. 2006. Tubbataha Reefs, a Marine Protected Area that Works, a Case Study on the Philippines. WWF-Philippines, Manila. 22 pp. (http:// wwf.org.ph/wwf3/downloads/publications/TubbatahaCaseStudy.pdf).

Estacion, J., V. Palaganas, R. Perez and M. Alava. 1993. Benthic characteristics of Islands and Reefs in the Sulu Sea, Philippines. Silliman Journal 36: 15-44.

Laureta, L.V. 2008. Compendium of the Economically Important Seashells in Panay, Philippines. The University of the Philippines Press, Quezon City. 147 pp.

Poppe, T.G. 2008a. Philippine Marine Mollusks. Vol. 1 (Gastropoda Part 1), Hackenheim: ConchBooks. 759 pp.

Poppe, T.G. 2008b. Philippine Marine Mollusks. Vol. 2 (Gastropoda Part 2), Hackenheim: ConchBooks. 848 pp.

Poppe, T.G. 2010a. Philippine Marine Mollusks. Vol. 3 (Gastropoda Part 3 and Bivalvia Part 1), Hackenheim: ConchBooks. 665 pp.

Poppe, T.G. 2010a. Philippine Marine Mollusks. Vol. 3 (Bivalvia - Part 2 and Scaphopoda, Polyplacophora, Cephalopdoa and Addenda), Hackenheim: ConchBooks. 675 pp.

Richer de Forges, B., S.H. Tan, P. Bouchet, Ng, P.k.L. and N. Saguil. 2009. Panglao 2005- Survey of the deep-water benthic fauna of the Bohol Sea and adjacent waters. The Raffles Bulletin of Zoology, Supplement 20: 21-38 (http://rmbr.nus.edu.sg/rbz/biblio/s2o/ s2orbzo21-038.pdf).

Songco, A.M. 2012. Field Guide to the Top Predators of the Tubbataha Reefs Natural Park, Cagayancillo, Palawan, Philippines. Puerto
Princesa: Pilipinas Shell Foundation. 42 pp

Springsteen, F.J. and F.M. Leobrera. 1986. Shells of the Philippines. Manila: Carfel Shell Museum. 377 pp.

Terryn, Y. 2007. A Collectors Guide to the Recent Terebridae (Mollusca: Neogastropoda). Hackenheim: ConchBooks. 57 pp.

TMO. 2013. Tubbataha Reefs Natural Park: Research and Monitoring Annual Report. Tubbataha Management Office, Puerto Princesa. $71 \mathrm{pp}$.

WoRMS. 2014. World Register of Marine Species. Accessible at http:// www.marinespecies.org. Captured on 8 August, 2014.

Yamaguchi, M. 1996. Shallow water molluscan assemblages of the Tubbataha Reef, Republic of the Philippines; pp. 61-71, in: Department of Environment and Natural Resources (DENR) and Marine Parks Center of Japan (MPCJ). The Report of the Project for Resources Survey and Conservation of Tubbataha Reefs National Marine Park. 200 pp.

Authors' contribution statement: RGD was responsible for photo-documentation, species identification and writing of the manuscript. RMP performed species identifications and prepared the figures. SFC did photo-documentation and species identification.

Recieved: June 2014

Accepted: December 2014

Editorial responsbility: Robert G. Forsyth 

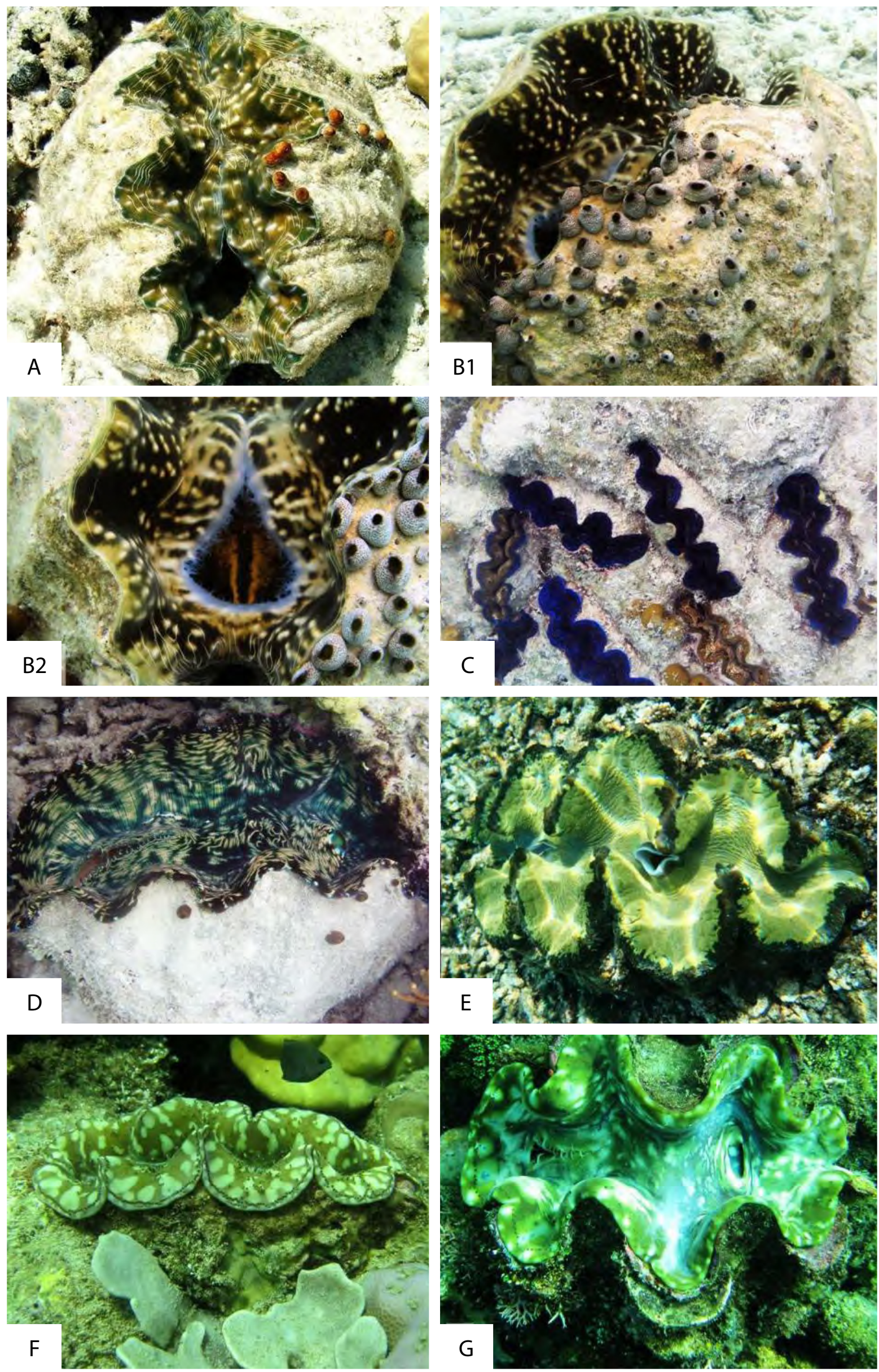

Figure 1. The giant clams of Tubbataha Reefs Natural Park. A) Hippopus hippopus, B1-2) Hippopus porcellanus, C) Tridacna crocea, D) Tridacna derasa, E) Tridacna gigas, F) Tridacna maxima, G) Tridacna squamosa. 


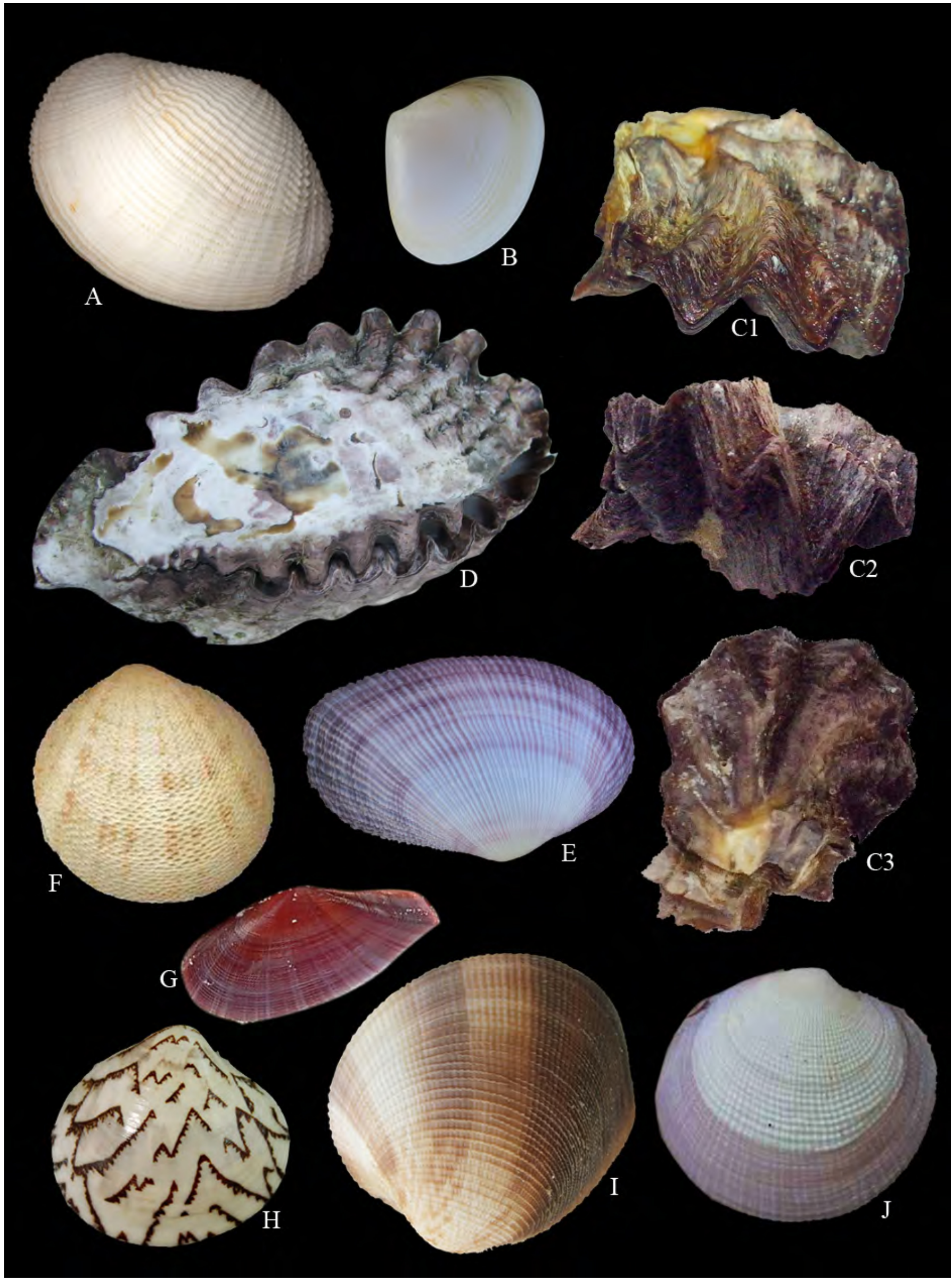

Figure 2. Some bivalve species from Tubbataha Reefs Natural Park. A) Fimbria fimbriata, B) Atactodea striata, C1-3) Lopha cristagalli, D) Saccostrea mordax, E) Asaphis violascens, F) Tellina scobinata, G) Tellina sp., H) Lioconcha castrensis, I) Periglypta puerpera, J) Codakia tigerina. 


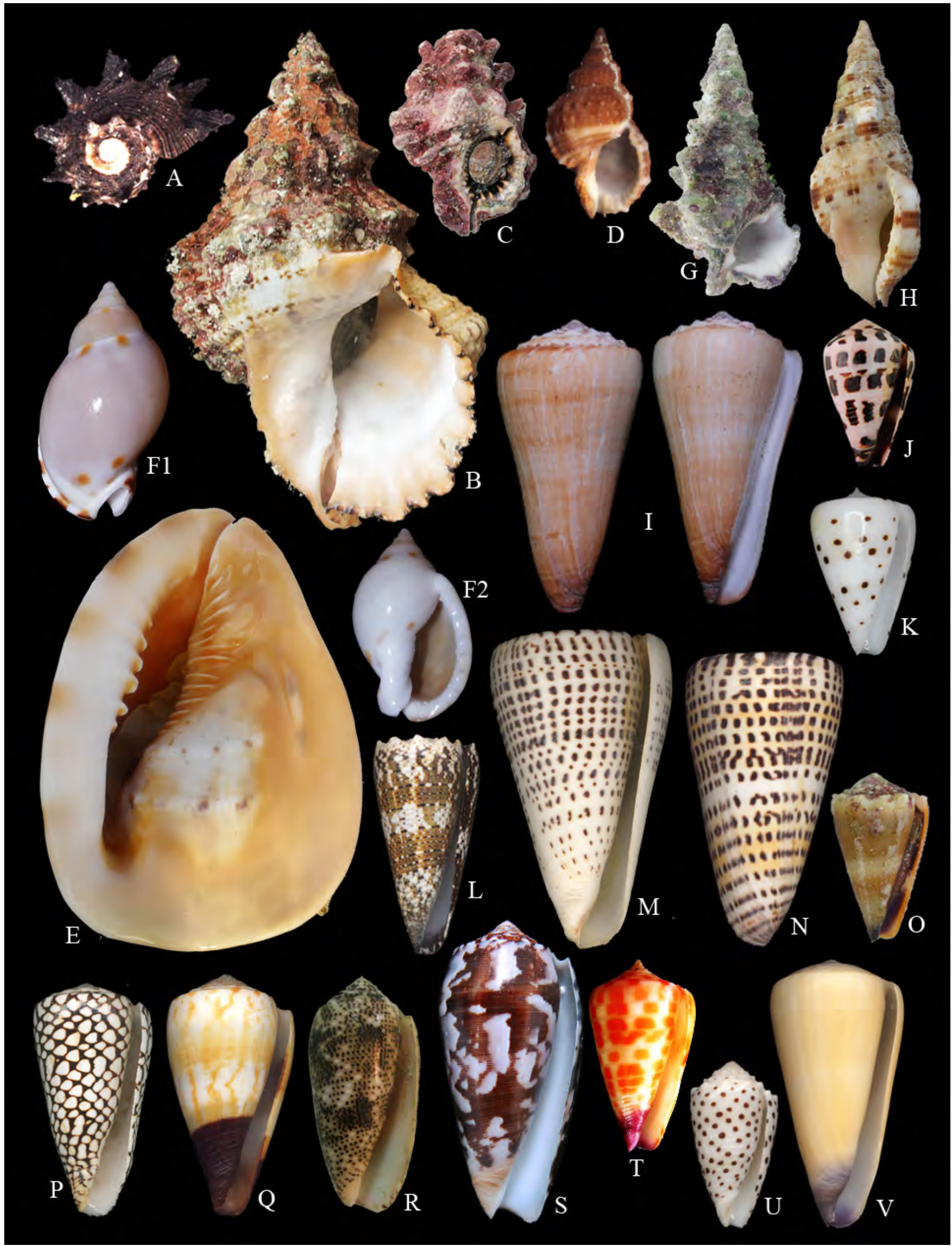

Figure 3. Some gastropod species at Tubbataha Reefs Natural Park. A) Angaria delphinus, B) Tutufa bubo, C) Bursa lamarckii, D) Bursa granularis, E) Cassis cornuta, F1-2) Casmaria ponderosa, G) Cerithium nodulosum, H) Colubraria muricata, I) Conus distans, J) Conus ebraeus, K) Conus eburneus, L) Conus imperialis, M) Conus leopardus, N) Conus litteratus, O) Conus lividus, P) Conus marmoreus, Q) Conus miles, R) Conus stercusmuscarum, S) Conus striatus, T) Conus tessulatus, U) Conus pulicarius, V) Conus virgo. 


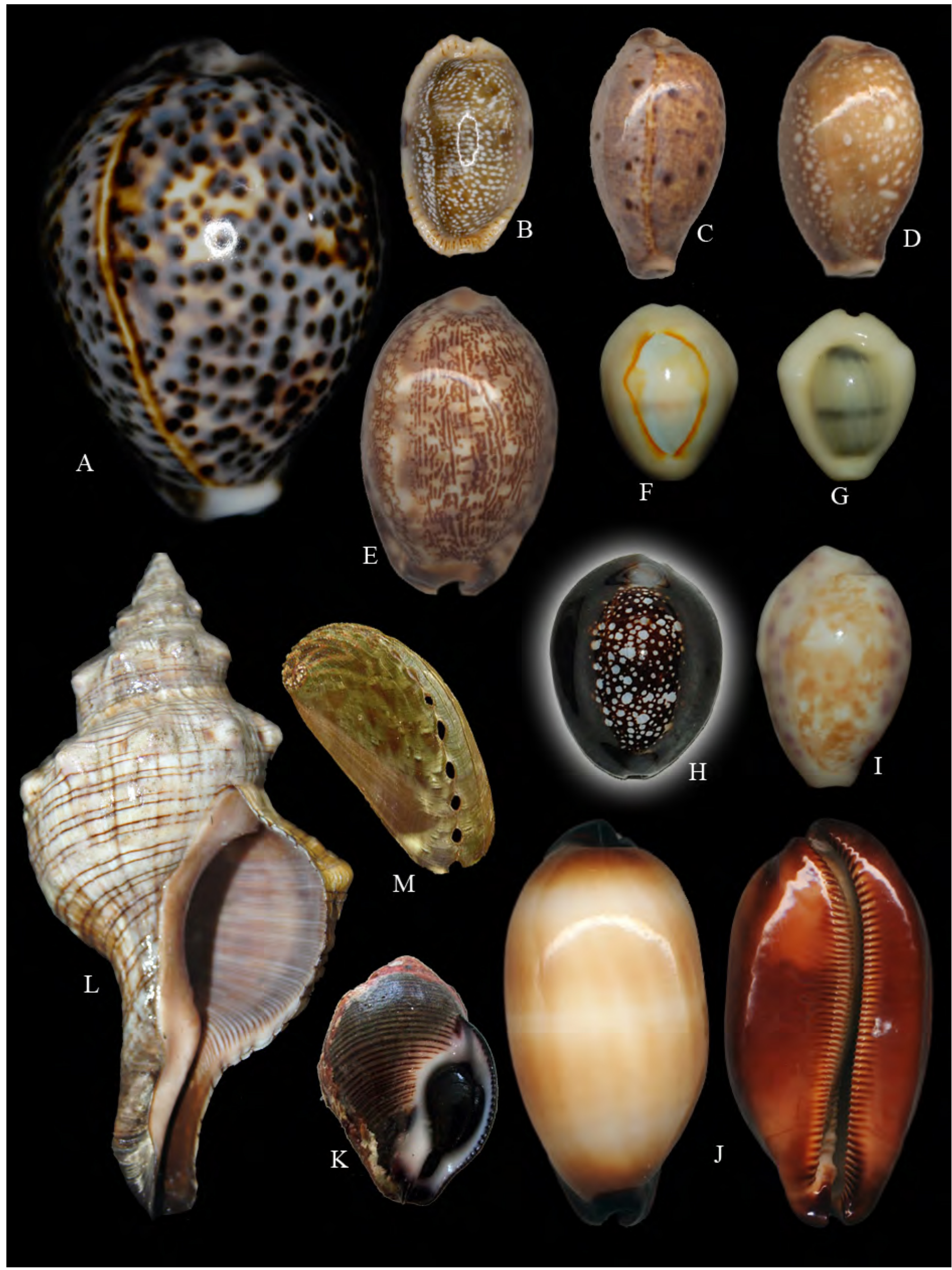

Figure 4. Some gastropod species at Tubbataha Reefs Natural Park. A) Cypraea tigris, B) Esoraria erosa, C) Lyncina lynx, D) Lyncina vitellus, E) Mauritia arabica, F) Monetaria annulus, G) Monetaria moneta, H) Monetaria caputserpentis, I) Ovatipsa chinensis, J) Talparia talpa, K) Latirolagena smaragdulus, L) Pleuroploca trapezium, M) Haliotis asinina. 


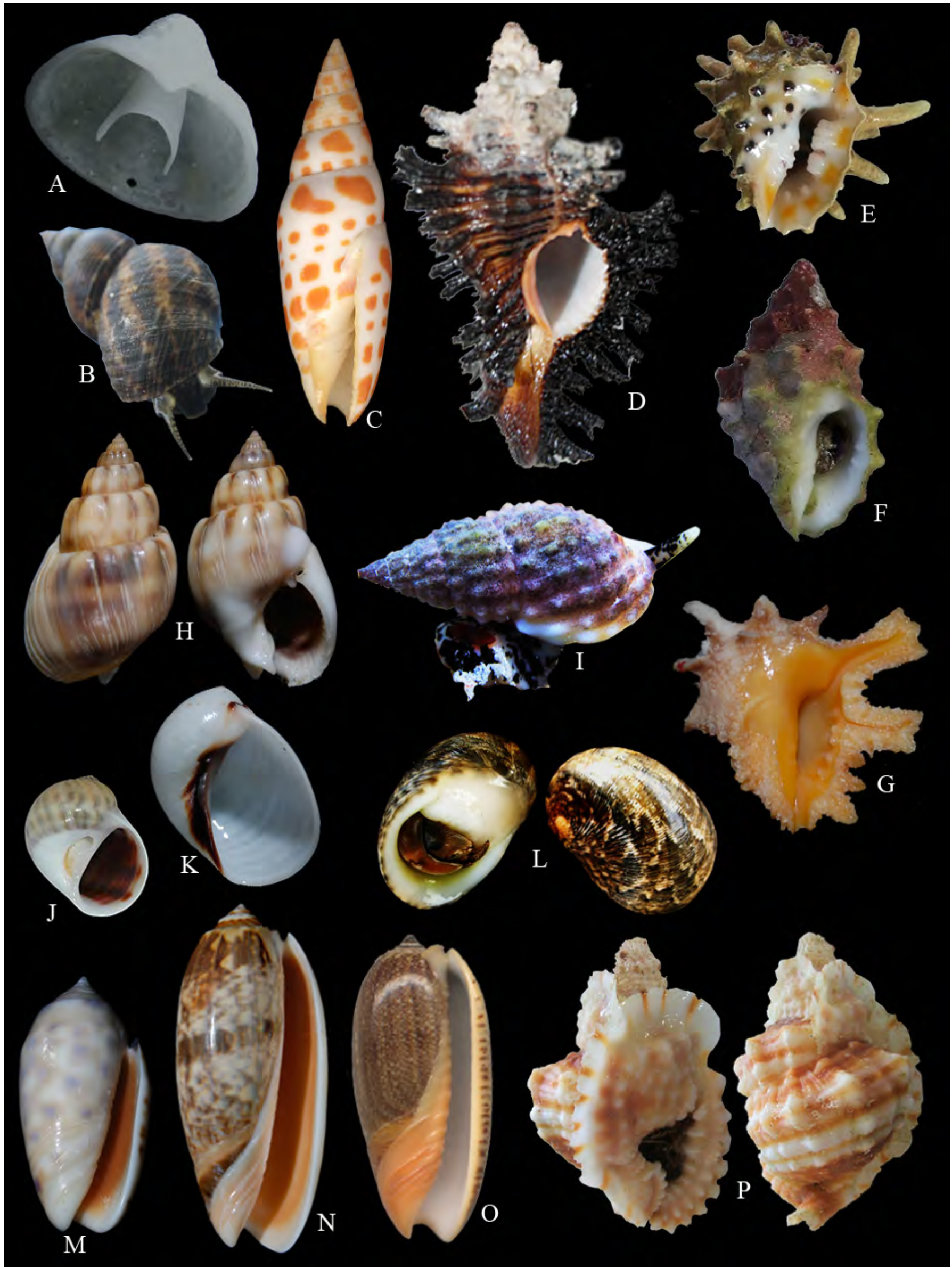

Figure 5. Some gastropod species from Tubbataha Reefs Natural Park. A) Cheilea cepacea, B) Littoraria scabra, C) Mitra mitra, D) Chicoreus brunneus, E) Drupa ricinus, F) Drupella cornus, G) Drupina grossularia, H) Nassarius coronatus, I) Nassarius papillosus, J) Notocochlis gualteriana, K) Mammilla sebae, L) Nerita polita, M) Oliva annulata, N) Oliva miniacea, O) Oliva reticulata, P) Distorsio anus. 


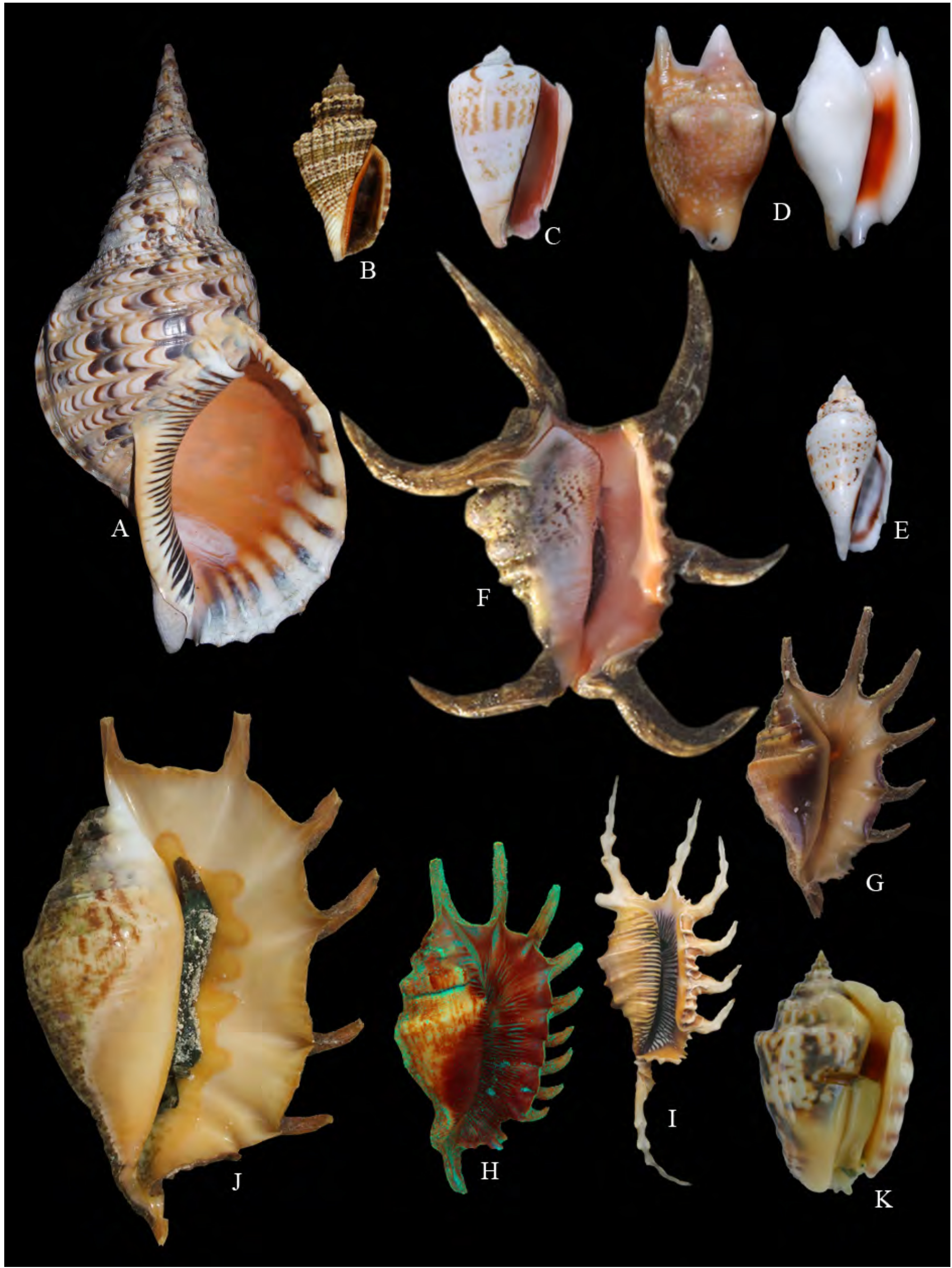

Figure 6. Some gastropod species from Tubbataha Reefs Natural Park. A) Charonia tritonis, B) Canarium labiatum, C) Conomurex luhuanus, D) Euprotomus bulla, E) Gibberulus gibberulus gibbosus, F) Harpago chiragra, G) Lambis lambis, H) Lambis millepeda, I) Lambis scorpius, J) Lambis sowerbyi, K) Lentigo lentiginosus. 


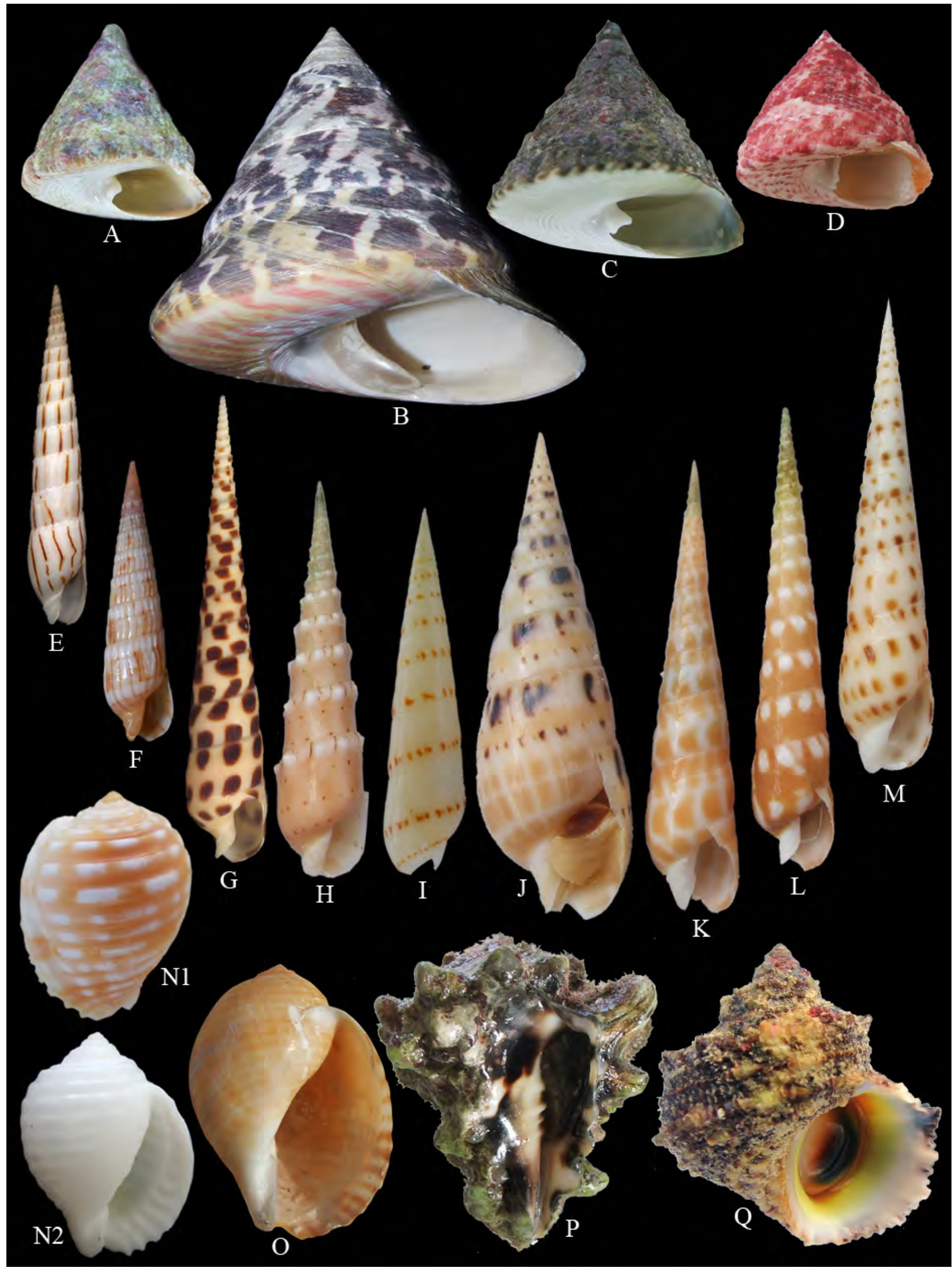

Figure 7. Some gastropod species from Tubbataha Reefs Natural Park. A) Tectus fenestratus, B) Tectus niloticus, C) Tectus pyramis, D) Trochus maculatus, E) Hastula lanceata, F) Myurella affinis, G) Terebra subulata, H) Oxymeris crenulata, I) Oxymeris felina, J) Oxymeris maculata, K) Oxymeris dimidiata, L) Terebra guttata, M) Oxymeris areolata, N1-2) Malea pomum, O) Tonna canaliculata, P) Vasum turbinellus, Q) Turbo chrysostomus. 